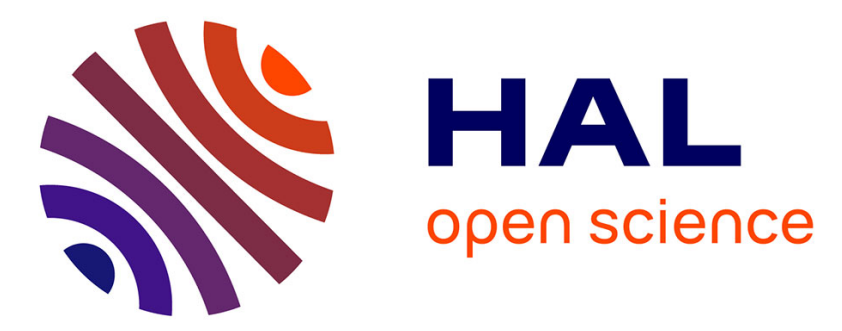

\title{
Variable selection and forecasting via automated methods for linear models: LASSO/adaLASSO and Autometrics
}

Camila Epprecht, Dominique Guegan, Álvaro Veiga, Joel Correa da Rosa

\section{To cite this version:}

Camila Epprecht, Dominique Guegan, Álvaro Veiga, Joel Correa da Rosa. Variable selection and forecasting via automated methods for linear models: LASSO/adaLASSO and Autometrics. 2017. halshs-00917797v2

\section{HAL Id: halshs-00917797 \\ https://shs.hal.science/halshs-00917797v2}

Submitted on 29 Dec 2017

HAL is a multi-disciplinary open access archive for the deposit and dissemination of scientific research documents, whether they are published or not. The documents may come from teaching and research institutions in France or abroad, or from public or private research centers.
L'archive ouverte pluridisciplinaire $\mathbf{H A L}$, est destinée au dépôt et à la diffusion de documents scientifiques de niveau recherche, publiés ou non, émanant des établissements d'enseignement et de recherche français ou étrangers, des laboratoires publics ou privés. 


\section{Documents de Travail du

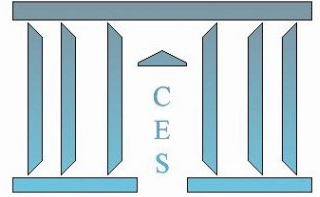

Variable selection and forecasting via automated methods for linear models: LASSO/adaLASSO and Autometrics

Camila EPPRECht, Dominique GUEGAn, Álvaro VeigA, Joel CORREA DA ROSA

\subsection{R}

Version révisée

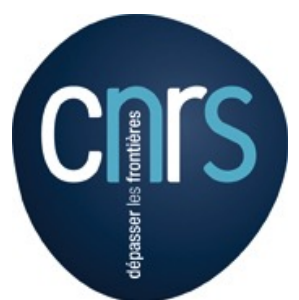




\title{
Variable selection and forecasting via automated methods for linear models: LASSO/adaLASSO and Autometrics
}

\author{
Camila Epprecht \\ Pontifical Catholic University of Rio de Janeiro, Rio de Janeiro, Brasil \\ Université Paris I Panthéon-Sorbonne, Paris, France \\ Dominique Guégan \\ Université Paris I Panthéon-Sorbonne, LabEx ReFi and IPAG, Paris, France \\ Álvaro Veiga \\ Pontifical Catholic University of Rio de Janeiro, Rio de Janeiro, Brasil \\ Joel Correa da Rosa \\ Icahn School of Medicine at Mount Sinai - Department of Population Health Science \& Policy, \\ New York, United States of America
}

\begin{abstract}
In this paper we compare two approaches of model selection methods for linear regression models: classical approach - Autometrics (automatic general-to-specific selection) - and statistical learning - LASSO ( $\ell_{1}$-norm regularization) and adaLASSO (adaptive LASSO). In a simulation experiment, considering a simple setup with orthogonal candidate variables and independent data, we compare the performance of the methods concerning predictive power (out-of-sample forecast), selection of the correct model (variable selection) and parameter estimation. The case where the number of candidate variables exceeds the number of observation is considered as well. Finally, in an application using genomic data from a highthroughput experiment we compare the predictive power of the methods to predict epidermal thickness in psoriatic patients.
\end{abstract}

Key Words: model selection, general-to-specific, adaptive LASSO, sparse models, Monte Carlo simulation, genetic data.

\section{Introduction}

The importance of automatic specification of statistical models has been growing exponentially with the progress and dissemination of data modeling. One important instance of this problem is the specification of multiple regression models. Presently, there are several statistical packages proposing different methodologies for selecting 
the explanatory variables from a set of candidates and estimating regression coefficients.

In this paper, we compare two of these methodologies among the most representatives of the two main approaches - traditional (Classical Approach) and shrinkage (Statistical Learning) - for this problem.

The Classical Approach uses mostly OLS, hypothesis testing and information criteria to compare different models. However, the total number of models to evaluate increases exponentially as the number of candidate variables increases. Moreover, the traditional OLS fails if the number of candidate variables is larger than the number of observations.

There are two main strategies to overcome combinatory problem of choosing the right set variables: specific-to-general and the general-to-specific. Some examples of specific-to-general methods are stepwise regression, forward selection and, the more recent, RETINA (Perez-Amaral et al., 2003) and QuickNet (White, 2006). In the general-to-specific (GETS) category the most important methods are based on a model selection strategy developed by the LSE school ('LSE' approach), revised in PcGets (Hendry and Krolzig, 1999, and Krolzig and Hendry, 2001), and more recently in Autometrics (Doornik, 2009), which will examined in this paper.

A competing approach - we will refer to it as 'shrinkage approach' - is mostly based on mathematical programming techniques and their conveniences. Those methods handle high dimensional data betting on sparsity, shrinking coefficients of irrelevant variables to zero during the estimation process. One of the first and most popular proposals of this type is the Least Absolute Shrinkage and Selection Operator (LASSO), introduced by Tibshirani (1996). A partial list of generalizations and adaptations of LASSO method to a variety of problems proposed in recent years can be found in Tibshirani (2011). Among these, the adaptive LASSO (adaLASSO), proposed by Zou (2006), has received particular attention.

Although the extensive recent literature in this field, no work has been done comparing Autometrics, which is a development of PcGets, with LASSO, or adaLASSO. These methods, based on two different approaches, have been broadly applied in the linear regression framework, for which their statistical properties have been already theoretically proven, as discussed in next section. Therefore, the aim of this paper is to compare selection and forecasting performances of these methods for linear regression models. In a simulation experiment we compare the predictive power 
(forecast out-of-sample) and the performance in the selection of the correct model and estimation (in-sample). The case where the number of candidate variables exceeds the number of observations is considered as well. In order to analyze different situations the model selection methodologies were compared varying the sample size, the number of relevant variables and the number of candidate variables. Finally, we apply the methods to predict psoriasis in a genetic study.

The paper is organized as follows. Model selection techniques are presented in Section 2. Section 3 presents the Monte Carlo experiment and simulation results. Section 4 is devoted to the application to epidermal thickness forecasting in psoriatic patients and results. Finally, Section 5 concludes.

\section{The model selection techniques}

\subsection{Autometrics}

The main pillar of this approach is the concept of GETS modeling: starting from a general dynamic statistical model which captures the main characteristics of the underlying data set, standard testing procedures are used to reduce its complexity by eliminating statistically insignificant variables, checking the validity of the reductions at every stage to ensure the congruence ${ }^{1}$ of the selected model.

Hendry and Krolzig (1999), and Krolzig and Hendry (2001) proposed an algorithm for automatic model selection, called PcGets. Using Monte Carlo simulation they studied the probabilities of PcGets recovering the data generating process (DGP), and they achieved good results. Campos et al. (2003) established the consistency of PcGets procedure.

Doornik (2009) introduced a third-generation algorithm, called Autometrics, based on the same principles. The new algorithm can also be applied in the general case of more variables than observations. Autometrics uses a tree-path search to detect and eliminate statistically insignificant variables. Such algorithm does not become stuck in a single-path, where a relevant variable is inadvertently eliminated, retaining other variables as proxies (e.g., as in stepwise regression).

\footnotetext{
${ }^{1}$ A congruent model should satisfy: (1) homoscedastic, independent errors; (2) strongly exogenous conditioning variables for the parameters of interest; (3) constant, invariant parameters of interest; (4) theory-consistent, identifiable structures; (5) data admissible formulations on accurate observations. For more details see Hendry and Nielsen (2007).
} 


\subsubsection{Methodology}

Autometrics has five basic stages: The first stage concerns the formulation of a linear model called the General Unrestricted Model (GUM); the second determines the estimation and testing of the GUM; the third is a pre-search process; the fourth is the tree-path search procedure; and the fifth is the selection of the final model.

The algorithm is described in detail in Doornik (2009). The main idea is to begin modeling with a linear model containing all candidate variables (GUM). The GUM is estimated by ordinary least squares and subjected to diagnostic tests. If there is statistically insignificant coefficient estimates, simpler models are estimated using a tree-path reduction search, and validated by diagnostic tests. If several terminal models are found, Autometrics tests them again their union. Rejected models are removed, and the union of the 'surviving' terminal models becomes a new GUM for another tree-path search iteration; then this entire search process continues and the terminal models are again tested against there union. If more than one model survives the encompassing tests, final choice is made by a pre-selected information criterion.

In the case where the number of candidate variables exceeds the number of observations, Autometrics applies the cross-block algorithm proposed in Hendry and Krolzig (2004), as described in the Appendix.

Autometrics is partially a black box. However, it allows the user to choose a number of settings to define modeling strategy, as the "target size" and the "tiebreaker". These will be briefly discussed in Section 3.

\subsection{LASSO and adaLASSO}

Shrinkage methods have become popular in the estimation of large dimensions models. Among these methods, the Least Absolute Shrinkage and Selection Operator (LASSO), proposed by Tibshirani (1996), has received particular attention because of the ability to shrink some parameters to zero, excluding irrelevant regressors. In other words, LASSO is a popular technique for simultaneous estimation and variable selection for linear models.

LASSO is able to handle more variables than observations and produces sparse models (Zhao and Yu, 2006, Meinshausen and Yu, 2009), which are easy to interpret. Moreover, the entire regularization path of the LASSO can be computed efficiently, 
as shown in Efron et al. (2004), or more recently in Friedman et al. (2010).

Despite all these nice characteristics, Zhao and $\mathrm{Yu}$ (2006) noted that the LASSO estimator can only be consistent if the design matrix ${ }^{2}$ satisfies a rather strong condition denoted "Irrepresentable Condition", which can be easily violated in the presence of highly correlated variables. Moreover, Zou (2006) noted that the oracle property in the sense of Fan and $\mathrm{Li}(2001)^{3}$ does not hold for LASSO. To amend these deficiencies, Zou (2006) proposes the adaptive LASSO (adaLASSO).

\subsubsection{The LASSO and adaLASSO estimators}

The LASSO technique is inspired in ridge regression, which is a standard technique for shrinking coefficients. However, contrarily to the latter, LASSO can set some coefficients to zero, resulting in an easily interpretable model.

Consider model estimation and variable selection in a linear regression framework. Suppose that $\mathbf{y}=\left(y_{1}, \ldots, y_{T}\right)^{\prime}$ is the response vector, and $\mathbf{x}_{j}=\left(x_{j 1}, \ldots, x_{j T}\right)^{\prime}$, with $j=1, \ldots, p$, are the predictor variables, possibly containing lags of $\mathbf{y}$.

The LASSO estimator, introduced by Tibshirani (1996), is given by

$$
\hat{\beta}^{\text {LASSO }}=\arg \min _{\beta}\left\|\mathbf{y}-\sum_{j=1}^{p} \mathbf{x}_{j} \beta_{j}\right\|^{2}+\lambda \sum_{j=1}^{p}\left|\beta_{j}\right|,
$$

where $\|$.$\| denotes the standard \ell^{2}$-norm, and $\lambda$ is a nonnegative regularization parameter. The second term in $(1)$ is the so-called " $\ell_{1}$ penalty", which is crucial for the success of the LASSO. The LASSO continuously shrinks the coefficients towards 0 as $\lambda$ increases, and some coefficients are shrunk to exact 0 if $\lambda$ is sufficiently large.

Zou (2006) showed the LASSO estimator does not enjoy the oracle property, and proposed a simple and effective solution, the adaptive LASSO, or adaLASSO. While in LASSO the coefficients are equally penalized in the $\ell_{1}$ penalty in the adaLASSO each coefficient is assign with different weights. Zou (2006) showed that

\footnotetext{
2 Design matrix: matrix of values of explanatory variables.

${ }^{3}$ Oracle property: the method both identifies the correct subset model and the estimates of non-zero parameters have the same asymptotic distribution as the ordinary least squares (OLS) estimator in a regression including only the relevant variables.
} 
if the weights are data-dependent and cleverly chosen, then the adaLASSO can have the oracle property.

The adaLASSO estimator is given by

$$
\hat{\beta}^{\text {adaLASSO }}=\arg \min _{\beta}\left\|\mathbf{y}-\sum_{j=1}^{p} \mathbf{x}_{j} \beta_{j}\right\|^{2}+\lambda \sum_{j=1}^{p} \widehat{w}_{j}\left|\beta_{j}\right|,
$$

where $\widehat{w}_{j}=1 /\left|\hat{\beta}_{j}^{*}\right|^{\gamma}, \gamma>0$, and $\hat{\beta}_{j}^{*}$ is an initial parameter estimate. As the sample size grows, the weights diverge (to infinity) for zero coefficients, whereas, for the non-zero coefficients, the weights converge to a finite constant. Zou (2006) suggests using the ordinary least squares (OLS) estimate of parameters as the initial parameter estimate $\hat{\beta}_{j}^{*}$. However, such estimator is not available when the number of candidate variables is larger than the number of observations. In this case, ridge regression can be used as an initial estimator. Recently, others estimators have been used as preestimators for adaLASSO. In their study, Medeiros and Mendes (2016) used elastic net procedure, proposed by Zou and Hastie (2005), as pre-estimator, showing good results for adaLASSO performance. Although we also tested OLS (when available), ridge and LASSO as pre-estimator, elastic net delivered the most robust results in our simulation exercise as well.

A critical point in LASSO and adaLASSO literature is the selection of the regularization parameter $\lambda$ and the weighting parameter $\gamma$. Traditionally, one employs cross-validation maximizing some predictive measure. However, using information criteria, such as Bayesian Information Criterion (BIC), has shown good results. Zou et al. (2007), Wang et al. (2007) and Zhang et al. (2010) studied such method. Wang et al. (2007) compared LASSO with tuning parameters selected by cross-validation and BIC, and showed that the LASSO with BIC selector performs better in the identification of the correct model. Furthermore, using BIC as selection criteria for the LASSO and adaLASSO performs remarkably well in Monte Carlo simulations presented in the Section 3.

\subsection{Theoretical comparison}

To compare the two approaches present in this paper, we focus on estimator bias and the average mean squared error (MSE). If the methodologies correctly select the true 
model, some results are expected concerning parameters estimates:

1. The MSE and bias of Autometrics estimators should be close to zero, as Autometrics model is the ordinary least squares (OLS) estimation for the selected final model.

2. LASSO and adaLASSO estimates should be smaller, in absolute values, than the population parameters, as they are shrinkage methods.

3. Bias of LASSO estimators tends to be larger in absolute value than the bias of adaLASSO estimators, which are expect to be close to the ones produced by OLS. The weighting strategy of adaLASSO makes the penalty term small for the relevant variables.

Therefore, in theory, when the true model is included, Autometrics is expected to be slightly superior to adaLASSO and a lot to LASSO, due to its oracle property. The differences between methods will appear in their variable selection performances.

\section{Simulation experiment}

We aim to analyze and compare variable selection and forecasting performance of Autometrics, LASSO and adaLASSO methods in different scenarios based on the same linear model, varying three parameters: numbers of observations, relevant variables and candidate variables. The scenarios and comparison statistics follow Medeiros and Mendes (2016).

The procedure used to solve LASSO is the glmnet package for Matlab, also used for ridge regression and elastic net. The glmnet procedure implements a coordinate descent algorithm. For more details, see Friedman et al. (2010). For Autometrics we used the procedure in OxMetrics package.

Regarding variable selection performance, our goal is to compare 'size' and 'power' of model selection process, namely the probability of inclusion in the final model of variables that do not (do) enter the DGP, i.e. retention frequency of irrelevant variables, and retention frequency of relevant variables.

We also analyze and compare the parameters estimates for each methodology. Finally, we compare the forecasting accuracy of models selected by each technique 
and by the Oracle model, which is the ordinary least squares (OLS) estimator in a regression including only the relevant variables.

To illustrate our purpose we chose to use a simple statistical model with orthogonal regressors and independent data, for which the compared methods have already proved to work well and their asymptotic properties have already been proven, as mentioned in Section 2. The data generating process (DGP) used is a Gaussian linear regression model, where the strongly exogenous variables are Gaussian whitenoise processes:

$$
\begin{aligned}
& y_{i}=\sum_{k=1}^{q} \beta_{k} x_{k, i}+\varepsilon_{i}, \quad \varepsilon_{i} \sim \mathrm{N}[0,0.01], \\
& \boldsymbol{x}_{i}=\boldsymbol{v}_{i}, \quad \boldsymbol{v}_{i} \sim \mathrm{N}_{q}\left[0, I_{q}\right] \text { for } i=1, \ldots, N,
\end{aligned}
$$

where $\boldsymbol{\beta}$ is a vector of ones of size $q$ and $\boldsymbol{x}_{i}$ is a vector of $q$ relevant variables.

The GUM is a linear regression model, which includes the intercept, the $q$ relevant variables of the DGP (3), and $p-q$ irrelevant variables, which are also Gaussian white-noise processes. The GUM, given by (4), has $p$ candidate variables and the constant,

$$
y_{i}=\pi_{0}+\sum_{k_{r}=1}^{q} \pi_{k_{r}} x_{k_{r}, i}+\sum_{k_{i}=1}^{p-q} \pi_{k_{i}} x_{k_{i}, i}+u_{i}, \quad u_{i} \sim \mathrm{N}\left[0, \sigma^{2}\right],
$$

where $k_{r}$ is the index of relevant variables and $k_{i}$ is the index of irrelevant variables.

We simulate $N=50,100,300,500$ observations of DGP (4) for different combinations of number of candidate $(p)$ and relevant $(q)$ variables. We consider $p=$ 100,300 and $q=5,10,15,20$. In other words, 32 different scenarios were evaluated in a Monte Carlo experiment with 1000 replications, combining parameters $N, p$ and $q$.

Models are estimated by Autometrics, LASSO and adaLASSO methods. The tuning parameters of LASSO and adaLASSO, $\lambda$ and $\gamma$, are selected by BIC, and elastic net is used as pre-estimator for adaLASSO. As to Autometrics, we compared two model strategies: Liberal and Conservative, i.e., "target size", which means "the proportion of irrelevant variables that survives the simplification process" (Doornik, 2009 ) was set to $5 \%$ (Liberal) and $1 \%$ (Conservative). For the final selection, BIC is used as “tie-breaker", and the rest of Autometrics's settings are defined by default. 


\subsection{Simulation results}

Results of the simulated scenarios are presented in Tables 1 to 6. For a descriptive statistics of the parameters estimates, Table 1 shows the average bias and the average mean squared error (MSE) for Autometrics (Liberal), Autometrics (Conservative), LASSO and adaLASSO estimators over the simulations and across candidate variables, i.e.,

$$
\begin{aligned}
\operatorname{Bias} & =\frac{1}{\mathrm{MC} * p} \sum_{i=1}^{p}\left(\sum_{j=1}^{\mathrm{MC}}\left(\hat{\beta}_{i, j}-\beta_{i, j}\right)\right) \\
\mathrm{MSE} & =\frac{1}{\mathrm{MC} * p} \sum_{i=1}^{p}\left(\sum_{j=1}^{\mathrm{MC}}\left(\hat{\beta}_{i, j}-\beta_{i, j}\right)^{2}\right),
\end{aligned}
$$

where

$$
\beta_{i, j}=\left\{\begin{array}{rr}
1, & \text { if } 1 \leq i \leq q \\
0, & \text { if } q+1 \leq i \leq p
\end{array} \quad, \forall j=1 \ldots \mathrm{MC},\right.
$$

is the vector of size $p$ of "true" values of the parameters of the model; $p$ and $q$ are the numbers of candidate and relevant variables, respectively; and $\mathrm{MC}$ is the number of Monte Carlo replications. In this experiment $\mathrm{MC}=1000$.

Table 1 presents very low variance (MSE) and bias in most of scenarios. This is explained by the large number of zero estimates as the table shows an average value across coefficients (relevant and irrelevant). Empirical results are in agreement with the theory in results 1 to 3 of Section 2.3, as well as the bias (absolute values) and MSE decrease with the sample size $(N)$. The bias of LASSO and adaLASSO are negative because the two shrinkage methods underestimate the $\beta$ 's that, in the simulation experiment, are positives. The bias (absolute values) and MSE of LASSO and adaLASSO estimators increase with $q$. When $p>N$, the average bias (absolute values) and variance of estimators increase, especially for LASSO and adaLASSO. However, the estimates are very precise in large samples, for all methods. 


\section{TABLE 1. PARAMETER ESTIMATES: DESCRIPTIVE STATISTICS}

Average bias and the average mean squared error (MSE), for each model selection technique, over all Monte Carlo simulations and parameter estimates, for each different sample size. $p$ is the number of candidate variables and $q$ is the number of relevant variables.

\begin{tabular}{|c|c|c|c|c|c|c|c|c|}
\hline \multirow[b]{2}{*}{$q \mid p$} & \multicolumn{2}{|c|}{$N=50$} & \multicolumn{2}{|c|}{$\underline{N=100}$} & \multicolumn{2}{|c|}{$N=300$} & \multicolumn{2}{|c|}{$N=500$} \\
\hline & 100 & 300 & 100 & 300 & 100 & 300 & 100 & 300 \\
\hline & \multicolumn{8}{|c|}{ average BIAS x $10^{-3}-$ Autometrics (Liberal) } \\
\hline 5 & 0.052 & 0.001 & -0.025 & -0.022 & 0.027 & 0.006 & -0.008 & 0.006 \\
\hline 10 & 0.007 & -0.008 & -0.007 & -0.011 & -0.010 & -0.006 & -0.007 & -0.002 \\
\hline 15 & 0.025 & 0.014 & 0.005 & 0.018 & -0.005 & -0.006 & -0.002 & 0.001 \\
\hline \multirow[t]{2}{*}{20} & -1.365 & -5.758 & 0.042 & -0.018 & -0.005 & 0.005 & 0.020 & 0.004 \\
\hline & \multicolumn{8}{|c|}{ average MSE x $10^{-3}$ - Autometrics (Liberal) } \\
\hline 5 & 0.205 & 0.055 & 0.061 & 0.076 & 0.011 & 0.017 & 0.007 & 0.006 \\
\hline 10 & 0.256 & 0.063 & 0.062 & 0.083 & 0.013 & 0.017 & 0.008 & 0.006 \\
\hline 15 & 0.322 & 0.072 & 0.073 & 0.088 & 0.015 & 0.018 & 0.009 & 0.006 \\
\hline \multirow[t]{2}{*}{20} & 3.052 & 10.892 & 0.086 & 0.093 & 0.016 & 0.018 & 0.009 & 0.007 \\
\hline & \multicolumn{8}{|c|}{ average BIAS x $10^{-3}-$ Autometrics (Conservative) } \\
\hline 5 & 0.020 & 0.003 & -0.013 & -0.001 & 0.013 & 0.003 & -0.003 & 0.004 \\
\hline 10 & 0.000 & -0.008 & 0.014 & -0.002 & 0.002 & -0.008 & -0.011 & -0.005 \\
\hline 15 & 0.009 & 0.008 & 0.006 & 0.006 & -0.010 & -0.004 & 0.001 & 0.002 \\
\hline \multirow[t]{2}{*}{20} & -4.973 & -3.047 & 0.014 & -0.009 & 0.005 & -0.002 & 0.018 & 0.000 \\
\hline & \multicolumn{8}{|c|}{ average MSE x $10^{-3}-$ Autometrics (Conservative) } \\
\hline 5 & 0.036 & 0.038 & 0.016 & 0.020 & 0.005 & 0.003 & 0.003 & 0.002 \\
\hline 10 & 0.059 & 0.055 & 0.020 & 0.015 & 0.007 & 0.004 & 0.004 & 0.003 \\
\hline 15 & 0.089 & 0.074 & 0.028 & 0.019 & 0.009 & 0.005 & 0.005 & 0.003 \\
\hline \multirow[t]{2}{*}{20} & 9.769 & 6.800 & 0.036 & 0.026 & 0.010 & 0.006 & 0.006 & 0.003 \\
\hline & \multicolumn{8}{|c|}{ average BIAS x $10^{-3}$ - LASSO } \\
\hline 5 & -1.561 & -0.716 & -1.141 & -0.476 & -0.648 & -0.263 & -0.516 & -0.204 \\
\hline 10 & -4.337 & -7.186 & -2.199 & -0.984 & -1.231 & -0.509 & -0.971 & -0.387 \\
\hline 15 & -16.571 & -30.851 & -3.961 & -1.763 & -2.813 & -0.958 & -2.720 & -0.910 \\
\hline \multirow[t]{2}{*}{20} & -66.433 & -47.215 & -6.150 & -3.191 & -4.547 & -1.536 & -4.420 & -1.476 \\
\hline & \multicolumn{8}{|c|}{ average MSE $\times 10^{-3}$ - LASSO } \\
\hline 5 & 0.079 & 0.048 & 0.035 & 0.017 & 0.011 & 0.005 & 0.007 & 0.003 \\
\hline 10 & 0.372 & 5.353 & 0.070 & 0.039 & 0.020 & 0.009 & 0.012 & 0.005 \\
\hline 15 & 8.476 & 34.590 & 0.147 & 0.088 & 0.061 & 0.021 & 0.054 & 0.018 \\
\hline \multirow[t]{2}{*}{20} & 66.917 & 58.235 & 0.276 & 0.290 & 0.117 & 0.040 & 0.105 & 0.035 \\
\hline & \multicolumn{8}{|c|}{ average BIAS x $10^{-3}$ - adaLASSO } \\
\hline 5 & -0.614 & -0.423 & $-0.3 \overline{15}$ & -0.194 & -0.302 & -0.103 & -0.302 & -0.099 \\
\hline 10 & -2.546 & -5.428 & -0.947 & -0.472 & -0.777 & -0.257 & -0.647 & -0.200 \\
\hline 15 & -12.710 & -24.434 & -2.749 & -0.914 & -2.654 & -0.883 & -2.573 & -0.816 \\
\hline \multirow[t]{2}{*}{20} & -58.327 & -38.909 & -4.289 & -1.606 & -4.329 & -1.455 & -4.245 & -1.361 \\
\hline & \multicolumn{8}{|c|}{ average MSE $\times 10^{-3}$ - adaLASSO } \\
\hline 5 & 0.138 & 0.295 & 0.008 & 0.004 & 0.004 & 0.001 & 0.003 & 0.001 \\
\hline 10 & 1.303 & 5.558 & 0.025 & 0.012 & 0.011 & 0.003 & 0.007 & 0.002 \\
\hline 15 & 9.914 & 29.961 & 0.089 & 0.030 & 0.058 & 0.019 & 0.051 & 0.016 \\
\hline 20 & 64.345 & 51.362 & 0.166 & 0.130 & 0.112 & 0.038 & 0.101 & 0.032 \\
\hline
\end{tabular}


Tables 2 to 5 present variable selection results for each model selection technique. Several related statistics are reported: Panel (a) presents the fraction of replications where the correct model has been selected, i.e., all relevant variables included and all irrelevant regressors excluded from the final model; Panel (b) shows the fraction of replications where the relevant variables are all included; Panel (c) presents the fraction of relevant variables included; Panel (d) shows the fraction of irrelevant variables excluded; Panel (e) presents the average number of included variables; Panel (f) shows the average number of included irrelevant variables. The following comments point out the main results in Tables 2 to 5:

1. adaLASSO presents the best performance in finding the correct sparsity pattern in most of the simulated scenarios. When $N=300$ and $N=500$, adaLASSO selects the correct model every time.

2. When $p>N$, LASSO and adaLASSO performance decreases dramatically as $q$ increases. In some extreme cases, adaLASSO includes more variables than observations.

3. Autometrics (Conservative) shows better performance than Autometrics (Liberal). As expected by definition of "target size", the former includes less irrelevant variables than the latter.

4. Autometrics (Conservative) shows best variable selection performance when $N=50$.

5. Performance of all methodologies improves with the sample size $(N)$ and gets worse as the number of candidate variables $(p)$ increases.

6. In most scenarios, performance of model selection methodologies gets worse as the number of relevant variables $(q)$ increases, especially when $p>N$. However, when $p<N$, LASSO and adaLASSO show an improvement in their performance for $q=15$ and $q=20$, explained by a feature of glmnet algorithm ${ }^{4}$.

Figure 1 shows the plot for Panel (a), (b), (c) and (c) of Tables 2 to 5: correct

\footnotetext{
${ }^{4}$ The glmnet algorithm estimates different models for a decreasing sequence of $\lambda$ 's. Values of $\lambda$ are data driven and the maximum $\lambda$ is the minimum value for which all coefficients estimates are zero. Different models are estimated for the entire sequence of $\lambda$ and we use the BIC for the final model selection. The glmnet algorithm has also stopping criteria that can reduce the number of estimated models. When $q=15$ and $q=20$ the algorithm do not estimate models for the entire sequence of $\lambda$ preventing the selection of over fitted models that minimize the BIC. For more details see glmnet vignette by Hastie, T. and Qian, J. (http://www.stanford.edu/ hastie/glmnet/glmnet_alpha.html).
} 
sparsity pattern, true model included, fraction of relevant variables included and fraction of irrelevant variables excluded. It is clear the superiority of adaLASSO to others model selection methods, except the case of $N=50$, where the best method is Autometrics (Conservative).

Finally, in order to compare predictive performance of the model selection methods, Table 6 reports the root mean squared error for out-of-sample forecasts (RMSFE) for Autometrics (Liberal and Conservative), LASSO, adaLASSO and Oracle models. We consider a total of 100 out-of-sample observations. Main results of Table 6 are summarized in the following comments:

1. As expected, all methodologies improve their performance as the sample size increases, and the number of relevant $(q)$ and candidate $(p)$ variables decreases.

2. When $p<N$ and $q$ is small, adaLASSO and Autometrics (Conservative) present similar performance to the Oracle model.

3. For $q=15$ or $q=20$, Autometrics (Conservative) presents lower RMSFE than adaLASSO, especially when $p>N$. 
TABLE 2. MODEL SELECTION: DESCRIPTIVE STATISTICS

Autometrics (Liberal)

Statistics concerning model selection for each different sample size. Panel (a) - fraction of replications where the correct model has been selected. Panel (b) - fraction of replications where the relevant variables are all included. Panel (c) - fraction of relevant variables included. Panel (d) - fraction of irrelevant variables excluded. Panel (e) - average number of included variables. Panel (f) - average number of included irrelevant variables. $p$ is the number of candidate variables and $q$ is the number of relevant variables.

\begin{tabular}{|c|c|c|c|c|c|c|c|c|}
\hline \multicolumn{9}{|c|}{ Autometrics (Liberal) } \\
\hline \multirow[b]{2}{*}{$q \mid p$} & \multicolumn{2}{|c|}{$N=50$} & \multicolumn{2}{|c|}{$N=100$} & \multicolumn{2}{|c|}{$N=300$} & \multicolumn{2}{|c|}{$N=500$} \\
\hline & 100 & 300 & 100 & 300 & 100 & 300 & 100 & 300 \\
\hline & \multicolumn{8}{|c|}{ Panel (a): Correct Sparsity Pattern } \\
\hline 5 & 0.011 & 0 & $0.0 \overline{18}$ & 0 & 0.006 & 0 & 0.008 & 0 \\
\hline 10 & 0.008 & 0 & 0.030 & 0 & 0.010 & 0 & 0.006 & 0 \\
\hline 15 & 0.006 & 0 & 0.033 & 0 & 0.004 & 0 & 0.007 & 0 \\
\hline 20 & 0.009 & 0 & 0.032 & 0 & 0.012 & 0 & 0.013 & 0 \\
\hline \multicolumn{9}{|c|}{ Panel (b): True Model Included } \\
\hline 5 & 1 & 1 & 1 & 1 & 1 & 1 & 1 & 1 \\
\hline 10 & 1 & 1 & 1 & 1 & 1 & 1 & 1 & 1 \\
\hline 15 & 1 & 1 & 1 & 1 & 1 & 1 & 1 & 1 \\
\hline 20 & 0.980 & 0.776 & 1 & 1 & 1 & 1 & 1 & 1 \\
\hline \multicolumn{9}{|c|}{ Panel (c): Fraction of Relevant Variables Included } \\
\hline 5 & 1 & 1 & 1 & 1 & 1 & 1 & 1 & 1 \\
\hline 10 & 1 & 1 & 1 & 1 & 1 & 1 & 1 & 1 \\
\hline 15 & 1 & 1 & 1 & 1 & 1 & 1 & 1 & 1 \\
\hline 20 & 0.994 & 0.922 & 1 & 1 & 1 & 1 & 1 & 1 \\
\hline \multicolumn{9}{|c|}{ Panel (d): Fraction of Irrelevant Variables Excluded } \\
\hline 5 & 0.820 & 0.883 & 0.910 & 0.769 & 0.950 & 0.912 & 0.948 & 0.958 \\
\hline 10 & 0.810 & 0.898 & 0.922 & 0.771 & 0.950 & 0.917 & 0.946 & 0.959 \\
\hline 15 & 0.812 & 0.914 & 0.923 & 0.780 & 0.946 & 0.918 & 0.946 & 0.958 \\
\hline 20 & 0.825 & 0.925 & 0.918 & 0.791 & 0.947 & 0.923 & 0.946 & 0.958 \\
\hline \multicolumn{9}{|c|}{ Panel (e): Number of Included Variables } \\
\hline 5 & 22.056 & 39.532 & 13.544 & 73.173 & 9.728 & 31.041 & 9.979 & 17.337 \\
\hline 10 & 27.103 & 39.577 & 17.026 & 76.438 & 14.542 & 34.139 & 14.870 & 21.981 \\
\hline 15 & 30.990 & 39.526 & 21.575 & 77.559 & 19.551 & 38.326 & 19.602 & 26.905 \\
\hline 20 & 33.882 & 39.419 & 26.595 & 78.381 & 24.227 & 41.518 & 24.306 & 31.846 \\
\hline \multicolumn{9}{|c|}{ Panel (f): Number of Included Irrelevant Variables } \\
\hline 5 & 17.056 & 34.532 & 8.544 & 68.173 & 4.728 & 26.041 & 4.979 & 12.337 \\
\hline 10 & 17.103 & 29.577 & 7.026 & 66.438 & 4.542 & 24.139 & 4.870 & 11.981 \\
\hline 15 & 15.990 & 24.526 & 6.575 & 62.559 & 4.551 & 23.326 & 4.602 & 11.905 \\
\hline 20 & 14.005 & 20.973 & 6.595 & 58.381 & 4.227 & 21.518 & 4.306 & 11.846 \\
\hline
\end{tabular}




\section{TABLE 3. MODEL SELECTION: DESCRIPTIVE STATISTICS Autometrics (Conservative)}

Statistics concerning model selection for each different sample size. Panel (a) - fraction of replications where the correct model has been selected. Panel (b) - fraction of replications where the relevant variables are all included. Panel (c) - fraction of relevant variables included. Panel (d) - fraction of irrelevant variables excluded. Panel (e) - average number of included variables. Panel (f) - average number of included irrelevant variables. $p$ is the number of candidate variables and $q$ is the number of relevant variables.

\begin{tabular}{|c|c|c|c|c|c|c|c|c|}
\hline \multicolumn{9}{|c|}{ "Autometrics (Conservative) } \\
\hline \multirow[b]{2}{*}{$q \mid p$} & \multicolumn{2}{|c|}{$N=50$} & \multicolumn{2}{|c|}{$N=100$} & \multicolumn{2}{|c|}{$N=300$} & \multicolumn{2}{|c|}{$N=500$} \\
\hline & 100 & 300 & 100 & 300 & 100 & 300 & 100 & 300 \\
\hline & \multicolumn{8}{|c|}{ Panel (a): Correct Sparsity Pattern } \\
\hline 5 & 0.425 & 0.091 & $0.4 \overline{47}$ & 0.115 & 0.357 & 0.201 & 0.341 & 0.075 \\
\hline 10 & 0.398 & 0.054 & 0.523 & 0.206 & 0.391 & 0.209 & 0.365 & 0.085 \\
\hline 15 & 0.369 & 0.029 & 0.513 & 0.182 & 0.378 & 0.163 & 0.384 & 0.068 \\
\hline \multirow[t]{2}{*}{20} & 0.368 & 0.023 & 0.529 & 0.144 & 0.430 & 0.147 & 0.413 & 0.073 \\
\hline & \multicolumn{8}{|c|}{ Panel (b): True Model Included } \\
\hline 5 & 1 & 1 & 1 & 1 & 1 & 1 & 1 & 1 \\
\hline 10 & 1 & 1 & 1 & 1 & 1 & 1 & 1 & 1 \\
\hline 15 & 1 & 1 & 1 & 1 & 1 & 1 & 1 & 1 \\
\hline \multirow[t]{2}{*}{20} & 0.911 & 0.839 & 1 & 1 & 1 & 1 & 1 & 1 \\
\hline & \multicolumn{8}{|c|}{ Panel (c): Fraction of Relevant Variables Included } \\
\hline 5 & 1 & 1 & 1 & 1 & 1 & 1 & 1 & 1 \\
\hline 10 & 1 & 1 & 1 & 1 & 1 & 1 & 1 & 1 \\
\hline 15 & 1 & 1 & 1 & 1 & 1 & 1 & 1 & 1 \\
\hline \multirow[t]{2}{*}{20} & 0.966 & 0.946 & 1 & 1 & 1 & 1 & 1 & 1 \\
\hline & \multicolumn{8}{|c|}{ Panel (d): Fraction of Irrelevant Variables Excluded } \\
\hline 5 & 0.986 & 0.966 & 0.987 & 0.976 & 0.988 & 0.991 & 0.988 & 0.989 \\
\hline 10 & 0.984 & 0.959 & 0.990 & 0.988 & 0.988 & 0.991 & 0.988 & 0.989 \\
\hline 15 & 0.983 & 0.953 & 0.990 & 0.987 & 0.987 & 0.989 & 0.988 & 0.989 \\
\hline \multirow[t]{2}{*}{20} & 0.982 & 0.955 & 0.990 & 0.985 & 0.988 & 0.989 & 0.988 & 0.989 \\
\hline & \multicolumn{8}{|c|}{ Panel (e): Number of Included Variables } \\
\hline 5 & 6.355 & 14.977 & 6.213 & 12.158 & 6.169 & 7.797 & 6.144 & 8.293 \\
\hline 10 & 11.401 & 21.911 & 10.883 & 13.476 & 11.088 & 12.753 & 11.037 & 13.125 \\
\hline 15 & 16.452 & 28.299 & 15.878 & 18.707 & 16.083 & 18.190 & 16.056 & 18.148 \\
\hline \multirow[t]{2}{*}{20} & 20.767 & 31.550 & 20.816 & 24.338 & 20.937 & 23.148 & 20.978 & 23.142 \\
\hline & \multicolumn{8}{|c|}{ Panel (f): Number of Included Irrelevant Variables } \\
\hline 5 & 1.355 & 9.977 & 1.213 & 7.158 & 1.169 & 2.797 & 1.144 & 3.293 \\
\hline 10 & 1.401 & 11.911 & 0.883 & 3.476 & 1.088 & 2.753 & 1.037 & 3.125 \\
\hline 15 & 1.452 & 13.299 & 0.878 & 3.707 & 1.083 & 3.190 & 1.056 & 3.148 \\
\hline 20 & 1.452 & 12.628 & 0.816 & 4.338 & 0.937 & 3.148 & 0.978 & 3.142 \\
\hline
\end{tabular}


TABLE 4. MODEL SELECTION: DESCRIPTIVE STATISTICS

LASSO

Statistics concerning model selection for each different sample size. Panel (a) - fraction of replications where the correct model has been selected. Panel (b) - fraction of replications where the relevant variables are all included. Panel (c) - fraction of relevant variables included. Panel (d) - fraction of irrelevant variables excluded. Panel (e) - average number of included variables. Panel (f) - average number of included irrelevant variables. $p$ is the number of candidate variables and $q$ is the number of relevant variables.

\begin{tabular}{|c|c|c|c|c|c|c|c|c|}
\hline \multicolumn{9}{|c|}{ LASSO } \\
\hline \multirow[b]{2}{*}{$q \mid p$} & \multicolumn{2}{|c|}{$N=50$} & \multicolumn{2}{|c|}{$N=100$} & \multicolumn{2}{|c|}{$N=300$} & \multicolumn{2}{|c|}{$N=500$} \\
\hline & 100 & 300 & 100 & 300 & 100 & 300 & 100 & 300 \\
\hline & \multicolumn{8}{|c|}{ Panel (a): Correct Sparsity Pattern } \\
\hline 5 & 0.015 & 0.007 & $0.0 \overline{76}$ & 0.056 & 0.199 & 0.147 & 0.285 & 0.205 \\
\hline 10 & 0.001 & 0 & 0.007 & 0.001 & 0.074 & 0.035 & 0.082 & 0.043 \\
\hline 15 & 0 & 0 & 0.003 & 0 & 0.401 & 0.146 & 0.865 & 0.664 \\
\hline \multirow[t]{2}{*}{20} & 0 & 0 & 0.001 & 0 & 0.522 & 0.172 & 0.936 & 0.828 \\
\hline & \multicolumn{8}{|c|}{ Panel (b): True Model Included } \\
\hline 5 & 1 & 1 & 1 & 1 & 1 & 1 & 1 & 1 \\
\hline 10 & 0.999 & 0.780 & 1 & 1 & 1 & 1 & 1 & 1 \\
\hline 15 & 0.937 & 0.049 & 1 & 1 & 1 & 1 & 1 & 1 \\
\hline \multirow[t]{2}{*}{20} & 0.378 & 0.002 & 1 & 1 & 1 & 1 & 1 & 1 \\
\hline & \multicolumn{8}{|c|}{ Panel (c): Fraction of Relevant Variables Included } \\
\hline 5 & 1 & 1 & 1 & 1 & 1 & 1 & 1 & 1 \\
\hline 10 & 1 & 0.963 & 1 & 1 & 1 & 1 & 1 & 1 \\
\hline 15 & 0.994 & 0.740 & 1 & 1 & 1 & 1 & 1 & 1 \\
\hline \multirow[t]{2}{*}{20} & 0.927 & 0.575 & 1 & 1 & 1 & 1 & 1 & 1 \\
\hline & \multicolumn{8}{|c|}{ Panel (d): Fraction of Irrelevant Variables Excluded } \\
\hline 5 & 0.918 & 0.960 & 0.957 & 0.984 & 0.976 & 0.992 & 0.981 & 0.993 \\
\hline 10 & 0.866 & 0.905 & 0.920 & 0.960 & 0.957 & 0.982 & 0.962 & 0.986 \\
\hline 15 & 0.780 & 0.862 & 0.904 & 0.938 & 0.986 & 0.989 & 0.998 & 0.998 \\
\hline \multirow[t]{2}{*}{20} & 0.663 & 0.859 & 0.885 & 0.906 & 0.991 & 0.992 & 0.999 & 0.999 \\
\hline & \multicolumn{8}{|c|}{ Panel (e): Number of Included Variables } \\
\hline 5 & 12.821 & 16.934 & 9.053 & 9.712 & 7.235 & 7.428 & 6.800 & 6.930 \\
\hline 10 & 22.038 & 37.173 & 17.164 & 21.532 & 13.866 & 15.243 & 13.383 & 14.049 \\
\hline 15 & 33.579 & 50.524 & 23.135 & 32.535 & 16.202 & 18.109 & 15.170 & 15.510 \\
\hline \multirow[t]{2}{*}{20} & 45.540 & 51.067 & 29.228 & 46.181 & 20.719 & 22.330 & 20.067 & 20.200 \\
\hline & \multicolumn{8}{|c|}{ Panel (f): Number of Included Irrelevant Variables } \\
\hline 5 & 7.821 & 11.934 & 4.053 & 4.712 & 2.235 & 2.428 & 1.800 & 1.930 \\
\hline 10 & 12.039 & 27.540 & 7.164 & 11.532 & 3.866 & 5.243 & 3.383 & 4.049 \\
\hline 15 & 18.674 & 39.429 & 8.135 & 17.535 & 1.202 & 3.109 & 0.170 & 0.510 \\
\hline 20 & 26.991 & 39.565 & 9.228 & 26.181 & 0.719 & 2.330 & 0.067 & 0.200 \\
\hline
\end{tabular}




\section{TABLE 5. MODEL SELECTION: DESCRIPTIVE STATISTICS adaLASSO}

Statistics concerning model selection for each different sample size. Panel (a) - fraction of replications where the correct model has been selected. Panel (b) - fraction of replications where the relevant variables are all included. Panel (c) - fraction of relevant variables included. Panel (d) - fraction of irrelevant variables excluded. Panel (e) - average number of included variables. Panel (f) - average number of included irrelevant variables. $p$ is the number of candidate variables and $q$ is the number of relevant variables.

\begin{tabular}{|c|c|c|c|c|c|c|c|c|}
\hline \multicolumn{9}{|c|}{ adaLASSO } \\
\hline \multirow[b]{2}{*}{$q \mid p$} & \multicolumn{2}{|c|}{$N=50$} & \multicolumn{2}{|c|}{$N=100$} & \multicolumn{2}{|c|}{$N=300$} & \multicolumn{2}{|c|}{$N=500$} \\
\hline & 100 & 300 & 100 & 300 & 100 & 300 & 100 & 300 \\
\hline & \multicolumn{8}{|c|}{ Panel (a): Correct Sparsity Pattern } \\
\hline 5 & 0.974 & 0.779 & 0.987 & 0.998 & 1 & 1 & 1 & 1 \\
\hline 10 & 0.901 & 0.316 & 0.982 & 0.995 & 1 & 1 & 1 & 1 \\
\hline 15 & 0.592 & 0.003 & 0.999 & 0.994 & 1 & 1 & 1 & 1 \\
\hline \multirow[t]{2}{*}{20} & 0.103 & 0.001 & 1 & 0.913 & 1 & 1 & 1 & 1 \\
\hline & \multicolumn{8}{|c|}{ Panel (b): True Model Included } \\
\hline 5 & 1 & 1 & 1 & 1 & 1 & 1 & 1 & 1 \\
\hline 10 & 0.999 & 0.789 & 1 & 1 & 1 & 1 & 1 & 1 \\
\hline 15 & 0.909 & 0.262 & 1 & 1 & 1 & 1 & 1 & 1 \\
\hline \multirow[t]{2}{*}{20} & 0.390 & 0.226 & 1 & 0.997 & 1 & 1 & 1 & 1 \\
\hline & \multicolumn{8}{|c|}{ Panel (c): Fraction of Relevant Variables Included } \\
\hline 5 & 1 & 1 & 1 & 1 & 1 & 1 & 1 & 1 \\
\hline 10 & 1 & 0.960 & 1 & 1 & 1 & 1 & 1 & 1 \\
\hline 15 & 0.989 & 0.762 & 1 & 1 & 1 & 1 & 1 & 1 \\
\hline \multirow[t]{2}{*}{20} & 0.905 & 0.628 & 1 & 1 & 1 & 1 & 1 & 1 \\
\hline & \multicolumn{8}{|c|}{ Panel (d): Fraction of Irrelevant Variables Excluded } \\
\hline 5 & 0.976 & 0.788 & 1 & 1 & 1 & 1 & 1 & 1 \\
\hline 10 & 0.927 & 0.725 & 1 & 1 & 1 & 1 & 1 & 1 \\
\hline 15 & 0.861 & 0.683 & 1 & 1 & 1 & 1 & 1 & 1 \\
\hline \multirow[t]{2}{*}{20} & 0.742 & 0.686 & 1 & 0.998 & 1 & 1 & 1 & 1 \\
\hline & \multicolumn{8}{|c|}{ Panel (e): Number of Included Variables } \\
\hline 5 & 7.256 & 67.577 & 5.050 & 5.019 & 5 & 5 & 5 & 5 \\
\hline 10 & 16.585 & 89.412 & 10.032 & 10.032 & 10 & 10 & 10 & 10 \\
\hline 15 & 26.648 & 101.668 & 15.001 & 15.017 & 15 & 15 & 15 & 15 \\
\hline \multirow[t]{2}{*}{20} & 38.750 & 100.427 & 20.000 & 20.623 & 20 & 20 & 20 & 20 \\
\hline & \multicolumn{8}{|c|}{ Panel (f): Number of Included Irrelevant Variables } \\
\hline 5 & 2.256 & 62.577 & 0.050 & 0.019 & 0 & 0 & 0 & 0 \\
\hline 10 & 6.586 & 79.813 & 0.032 & 0.032 & 0 & 0 & 0 & 0 \\
\hline 15 & 11.813 & 90.234 & 0.001 & 0.017 & 0 & 0 & 0 & 0 \\
\hline 20 & 20.660 & 87.860 & 0 & 0.627 & 0 & 0 & 0 & 0 \\
\hline
\end{tabular}




\section{FIGURE 1. MODEL SELECTION: COMPARISON}

Panel (a), (b), (c) and (d) for Autometrics-Lib (red), Autometrics-Cons (yellow), LASSO (green) and adaLASSO (blue). $p$ is the number of candidate variables, $q$ is the number of relevant regressors and $N$ is the sample size.
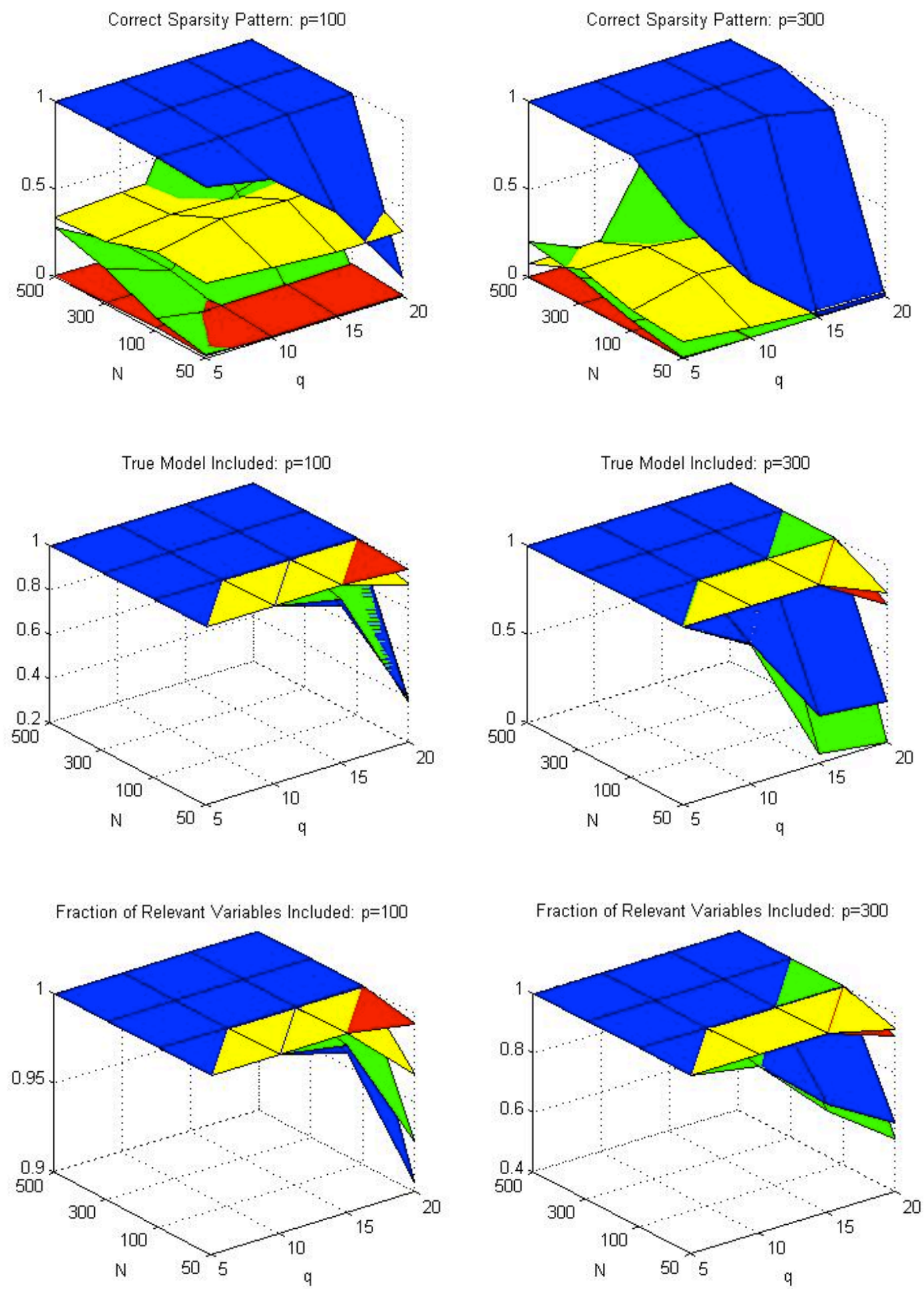

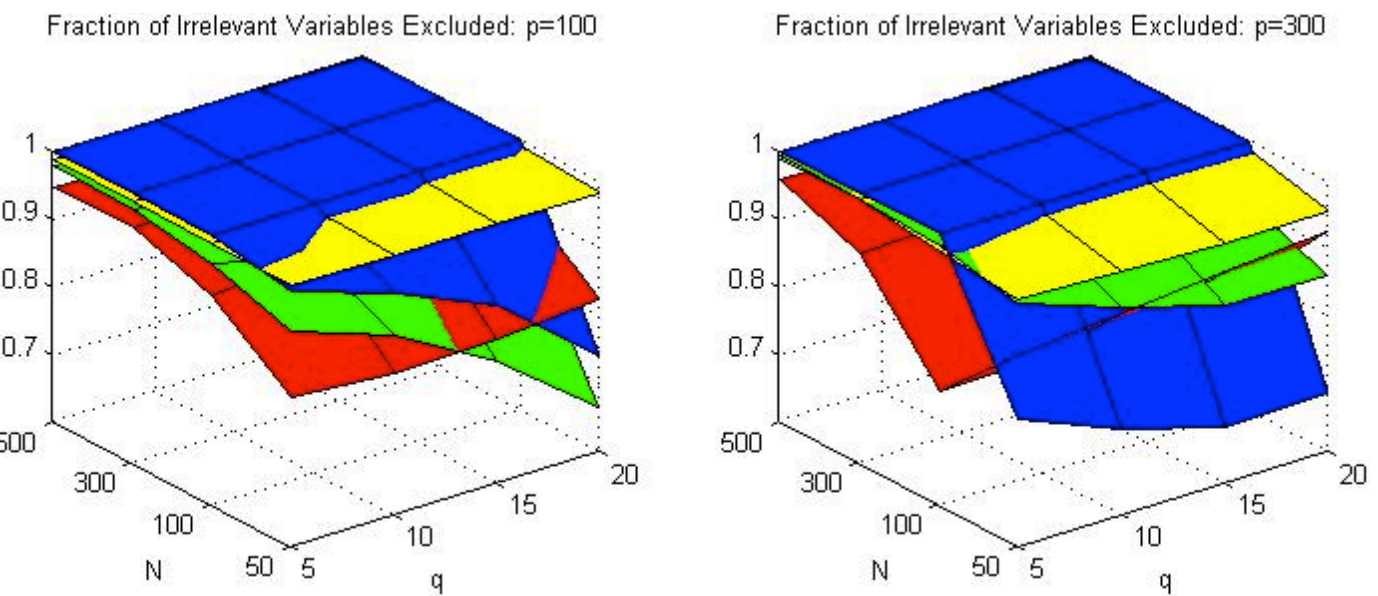

TABLE 6. FORECASTING: RMSFE

Root mean squared forecast error (RMSFE), for each model selection technique, for each different sample size. $p$ is the number of candidate variables and $q$ is the number of relevant variables.

\begin{tabular}{|c|c|c|c|c|c|c|c|c|}
\hline \multirow[b]{2}{*}{$q \mid p$} & \multicolumn{2}{|c|}{$N=50$} & \multicolumn{2}{|c|}{$N=100$} & \multicolumn{2}{|c|}{$N=300$} & \multicolumn{2}{|c|}{$N=500$} \\
\hline & 100 & 300 & 100 & 300 & 100 & 300 & 100 & 300 \\
\hline & \multicolumn{8}{|c|}{ RMSFE - Autometrics (Liberal) } \\
\hline 5 & 0.172 & 0.163 & 0.126 & 0.181 & 0.105 & 0.123 & 0.103 & 0.108 \\
\hline 10 & 0.186 & 0.170 & 0.127 & 0.186 & 0.106 & 0.122 & 0.104 & 0.108 \\
\hline 15 & 0.203 & 0.177 & 0.130 & 0.190 & 0.107 & 0.123 & 0.104 & 0.109 \\
\hline \multirow[t]{2}{*}{20} & 0.285 & 1.002 & 0.136 & 0.195 & 0.107 & 0.123 & 0.105 & 0.110 \\
\hline & \multicolumn{8}{|c|}{ RMSFE - Autometrics (Conservative) } \\
\hline 5 & 0.116 & 0.145 & 0.108 & 0.126 & 0.102 & 0.105 & 0.101 & 0.103 \\
\hline 10 & 0.125 & 0.165 & 0.110 & 0.119 & 0.103 & 0.106 & 0.102 & 0.103 \\
\hline 15 & 0.137 & 0.178 & 0.113 & 0.125 & 0.104 & 0.107 & 0.102 & 0.104 \\
\hline \multirow[t]{2}{*}{20} & 0.427 & 0.737 & 0.116 & 0.132 & 0.105 & 0.108 & 0.103 & 0.105 \\
\hline & \multicolumn{8}{|c|}{ RMSFE - LASSO } \\
\hline 5 & 0.133 & 0.155 & 0.116 & 0.123 & 0.105 & 0.107 & 0.103 & 0.104 \\
\hline 10 & 0.202 & 0.875 & 0.130 & 0.147 & 0.110 & 0.113 & 0.105 & 0.107 \\
\hline 15 & 0.628 & 3.164 & 0.157 & 0.190 & 0.127 & 0.127 & 0.124 & 0.124 \\
\hline \multirow[t]{2}{*}{20} & 2.330 & 4.173 & 0.193 & 0.280 & 0.147 & 0.148 & 0.143 & 0.143 \\
\hline & \multicolumn{8}{|c|}{ RMSFE - adaLASSO } \\
\hline 5 & 0.123 & 0.216 & 0.104 & 0.106 & 0.102 & 0.102 & 0.101 & 0.101 \\
\hline 10 & 0.219 & 0.927 & 0.112 & 0.117 & 0.105 & 0.105 & 0.103 & 0.103 \\
\hline 15 & 0.609 & 2.892 & 0.137 & 0.137 & 0.125 & 0.125 & 0.122 & 0.122 \\
\hline \multirow[t]{2}{*}{20} & 2.219 & 3.858 & 0.161 & 0.175 & 0.145 & 0.146 & 0.141 & 0.140 \\
\hline & \multicolumn{8}{|c|}{ RMSFE - Oracle } \\
\hline 5 & 0.105 & 0.105 & 0.103 & 0.103 & 0.101 & 0.101 & 0.100 & 0.100 \\
\hline 10 & 0.112 & 0.112 & 0.105 & 0.105 & 0.102 & 0.102 & 0.101 & 0.101 \\
\hline 15 & 0.120 & 0.120 & 0.108 & 0.108 & 0.102 & 0.102 & 0.101 & 0.102 \\
\hline 20 & 0.129 & 0.130 & 0.112 & 0.112 & 0.103 & 0.103 & 0.102 & 0.102 \\
\hline
\end{tabular}




\section{Application: Epidermal thickness in psoriatic patients}

Psoriasis is a common chronic inflammatory skin disease, which the cause is not entirely understood. Clinically, thickened epidermis is a major factor to measure psoriasis severity.

With recent evolution of high-throughput technologies devoted to medical and translational sciences, genomics databases are increasingly available, and the development of high-dimensional statistical models becomes essential. In this scenario, variable selection is a significant step, and some methodologies have already been applied to genomics. Tian et al. (2012), Tian and Suárez-Fariñas (2013) and Correa da Rosa et al. (2017) show applications of regularization algorithms for genes selection in Psoriasis' genomic data.

A set of histological measurements of epidermal thickness in a cohort of 609 psoriatic patients reported in Suárez-Fariñas et al. (2012) $)^{5}$ and a subcohort of 65 patients in Kim et al. (2015) were analysed and showed evidence of association between gene expression levels and thick and thin plaque psoriasis phenotypes. Despite the fact that the authors have identified psoriasis pathways with difference between these two phenotypes, the quantitative epidermal thickness phenotype was not used as an outcome. Additionally, enrichment analysis was only carried out for psoriasis pathways.

In this section, we propose fill these gaps with the application of the two described approaches. The epidermal thickness and microarray gene expressions from 54675 genes measured in a set of 70 patients analysed in Suárez-Fariñas et al. (2012) will be used as dependent and regressors respectively. Due to the fact that Autometrics does not support a General Unrestricted Model (GUM) with such a large number of candidate variables, we used only a restricted set of 870 gene expressions ${ }^{6}$ as regressors. The GUM is a linear model written in eq. (8).

$$
\begin{gathered}
y_{i}=\sum_{k=1}^{p} \beta_{k} x_{k, i}+\varepsilon_{i}, \quad i=1, \ldots, N \\
\varepsilon_{i} \sim \operatorname{IN}\left[0, \sigma^{2}\right]
\end{gathered}
$$

\footnotetext{
${ }^{5}$ We want to thank Mayte Suárez-Fariñas from the Icahn School of Medicine, Mount Sinai, New York, USA, for providing the data and all the help with the genetic language.

${ }^{6} \mathrm{An}$ initial set of 54675 candidate variables (microarray gene expressions at probesets) was reduced to a set of 870 genes by using moderated t-test statistics in linear mixed-effects models as implemented in limma package available in $\mathrm{R} /$ Bioconductor software.
} 
where $p$ is 870 candidate variables.

We used $80 \%$ of the data for specification and estimation in-sample (56 patients) and the final $20 \%$ for out-of-sample forecasting (14 patients). We evaluated 1000 permutations on the data observations, creating 1000 different in-sample and out-of-sample sets. The results presented next are the average statistics of the 1000 fitted models.

\subsection{Results}

We considered GUM in eq. (8) for specification and estimation by Autometrics, LASSO and adaLASSO methods, and evaluate one-step ahead out-of-sample forecast.

Out-of-sample forecasting is evaluated in terms of two measures: root mean squared forecast error (RMSFE) and an out-of-sample $R^{2}$ statistics, defined as:

$$
R_{o s}^{2}=1-\sum_{t \in \mathrm{O}} \frac{\left(y_{t}-\hat{y}_{t}\right)^{2}}{\left(y_{t}-\bar{y}\right)^{2}},
$$

where 0 is the out-of-sample observations set and $\bar{y}$ is the historical mean of the insample set. Contrarily to usual $R^{2}$, the out-of-sample $R^{2}$ may be negative. If $R^{2}{ }_{o S}$ is positive, then the selected model has lower average mean squared prediction error than the historical average.

Table 7 presents results concerning estimation (in-sample) and forecasting (outof-sample) for model selection methods. With respect to variable selection and estimation, Table 7 reports the average number of parameters and the average insample $R^{2}$, for selected final models. Concerning one-step ahead out-of-sample forecasting, Table 7 presents the average root mean squared forecast error (RMSFE), and average out-of-sample $R^{2}$, defined in eq. (9). The following comments point out the main results:

1. The out-of-sample forecasting performance of LASSO and adaLASSO models is far superior to Autometrics (Liberal and Conservative) models.

2. The LASSO model presents the best predictive performance, i.e., the lowest RMSFE and largest $R^{2}$ os. 


\section{TABLE 7. PSORIASIS FORECASTING: ACCURACY STATISTICS}

Estimation (in-sample) and forecasting (out-of-sample) accuracy average measures for each model selection technique: number of parameters; in-sample $R^{2}$; one-step ahead root mean squared forecast error (RMSFE), and out-of-sample $R^{2}$.

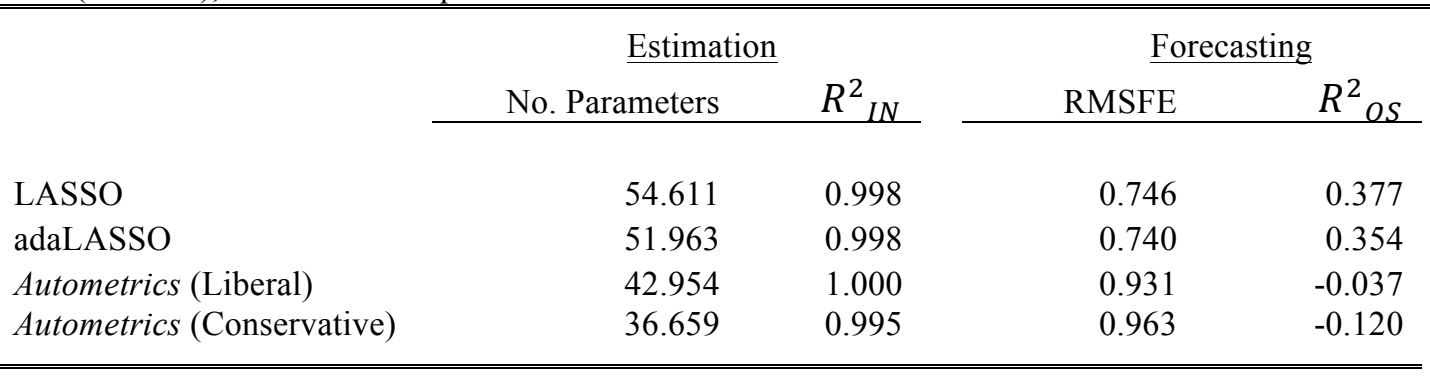

In order to measure the statistical significance of the differences between the forecast errors of the tested models we employ the modified Diebold-Mariano test, a more robust version, proposed by Harvey et al. (1997). We apply the test with different functions for the out-of-sample forecast: absolute error and squared error. We tested the null hypothesis of "equal accuracy" of models with a reference.

The test statistics and the p-value are presented in Table 8 . The test shows that LASSO and adaLASSO present out-of-sample absolute (p-values $10.5 \%$ and $10.1 \%$ ) and squared errors (p-values 13.6\% and 13.2\%) significantly lower than Autometrics (Conservative), and at a significant level of $13.2 \%$ and $12.5 \%$, respectively, present out-of-sample absolute error lower than Autometrics (Liberal). We can say that adaLASSO and LASSO have more predictive power than Autometrics.

\section{TABLE 8. TEST OF PREDICTIVE ACCURACY}

The table reports the modify Diebold and Mariano test statistic and p-values (in bracket) for all models, for absolute error and squared error. Models in columns are compared with models in rows (reference).

\begin{tabular}{|c|c|c|c|c|c|c|}
\hline & \multicolumn{3}{|c|}{ absolute error } & \multicolumn{3}{|c|}{$\underline{\text { squared error }}$} \\
\hline & adaLASSO & Aut (lib) & Aut (cons) & adaLASSO & Aut (lib) & Aut (cons) \\
\hline LASSO & $\begin{array}{c}0.208 \\
(0.419)\end{array}$ & $\begin{array}{l}-1.166 \\
(0.132)\end{array}$ & $\begin{array}{l}-1.316 \\
(0.105)\end{array}$ & $\begin{array}{c}0.245 \\
(0.405)\end{array}$ & $\begin{array}{c}-0.986 \\
(0.171)\end{array}$ & $\begin{array}{l}-1.145 \\
(0.136)\end{array}$ \\
\hline adaLASSO & - & $\begin{array}{l}-1.202 \\
(0.125)\end{array}$ & $\begin{array}{c}-1.341 \\
(0.101)\end{array}$ & - & $\begin{array}{c}-1.028 \\
(0.161)\end{array}$ & $\begin{array}{l}-1.170 \\
(0.132)\end{array}$ \\
\hline Aut (lib) & - & - & $\begin{array}{l}-0.147 \\
(0.443)\end{array}$ & - & - & $\begin{array}{l}-0.130 \\
(0.449)\end{array}$ \\
\hline
\end{tabular}

Each fitted model provided us a list of genes ranked by the frequency of selection. Biological validation of these lists was performed with Gene Set Enrichment Analysis (GSEA) run on a set of 257 Psoriasis pathways curated by the 
Laboratory of Investigative Dermatology at The Rockefeller University. Table 9 shows the top 3-associated psoriasis' gene sets in each fitted model as well as the pvalue adjusted by the Benjamini-Hochberg method (FDR).

Table 9 shows concordance in the top association for LASSO and adaLASSO and the same happened for Autometric's algorithms. The Autometrics Liberal and Conservative have generated ranked gene lists that are significantly enriched in the up-regulated genes found in Bowcock (2001). This result agrees with one of the pathways reported in Kim (2015) when analyzing differences in thick and thin psoriasis.

\section{TABLE 9. BIOLOGICAL VALIDATION OF RANKED GENES}

Top 3-associated Gene Sets with ranked lists of genes obtained by the different fitted models.

\begin{tabular}{|c|c|c|c|c|}
\hline & LASSO & adaLASSO & Autometrics (Lib) & Autometrics (Cons) \\
\hline (1) & $\begin{array}{l}\text { Genes down- } \\
\text { regulated in BCC } \\
\text { and Kaposi's } \\
\text { sarcoma }\left(\mathrm{p}=0.004^{*}\right)\end{array}$ & $\begin{array}{l}\text { Genes down- } \\
\text { regulated in } \\
\text { psoriasis detected by } \\
\text { NGS }(p=0.136)\end{array}$ & $\begin{array}{l}\text { Genes up-regulated } \\
\text { in Psoriasis by } \\
\text { Bowcock }(2001) \\
\left(p=0.026^{*}\right)\end{array}$ & $\begin{array}{l}\text { Genes up-regulated } \\
\text { in Psoriasis by } \\
\text { Bowcock }(2001) \\
\left(\mathrm{p}=0.003^{*}\right)\end{array}$ \\
\hline (2) & $\begin{array}{l}\text { Genes down- } \\
\text { regulated in } \\
\text { psoriasis detected by } \\
\text { NGS }(\mathrm{p}=0.118)\end{array}$ & $\begin{array}{l}\text { Genes down- } \\
\text { regulated in BCC } \\
\text { and Kaposi's } \\
\text { sarcoma }(p=1.000)\end{array}$ & $\begin{array}{l}\text { Genes up-regulated } \\
\text { in Atopic Dermatitis } \\
\text { lesional skin vs. } \\
\text { non-lesional skin } \\
(p=0.548)\end{array}$ & $\begin{array}{l}\text { IL-17 and TNF-a } \\
\text { additive effect in } \\
\text { keratinocytes } \\
(p=0.416)\end{array}$ \\
\hline (3) & $\begin{array}{l}\text { Genes down- } \\
\text { regulated in } \\
\text { Psoriasis by } \\
\text { Bowcock }(2001) \\
(p=0.742)\end{array}$ & $\begin{array}{l}\text { Genes down- } \\
\text { regulated in MPH } \\
\text { LPS and IFNg } \\
(p=1.000)\end{array}$ & $\begin{array}{l}\text { Genes down- } \\
\text { regulated in } \\
\text { Keratinocytes and } \\
\text { IFNg in Swindell } \\
(p=0.674)\end{array}$ & $\begin{array}{l}\text { Genes down- } \\
\text { regulated in } \\
\text { Keratinocytes and } \\
\text { IFNg in Swindell } \\
(p=0.511)\end{array}$ \\
\hline
\end{tabular}

\section{Conclusions}

In this paper we compare two approaches for model selection considering different aspects and scenarios: Autometrics, using Liberal and Conservative strategies, and LASSO/adaLASSO.

Considering a very simple setup, we conduct a Monte Carlo simulation experiment where the DGP is a linear regression with orthogonal variables and independent data. Three aspects of the performance are considered: variable selection, 
parameter estimation and predictive power, considering different sample sizes $(N)$, different number of relevant variables $(q)$ and candidate variables $(p)$. Simulation results show that, as expected, all methods improve their performance as sample size increases and the number of relevant and candidate variables decreases. Regarding parameter estimation, Autometrics presents the lowest absolute average bias and variance, as expected by the definition of OLS estimation when the correct model is selected. LASSO and adaLASSO present similar results when $N$ increases, however, for small sample sizes, adaLASSO presents lower parameters average absolute bias and variance.

Regarding variable selection, adaLASSO presents superior performance in most of the simulated scenarios, except for $N=50$, where Autometrics (Conservative) presents better results, especially if the number of relevant variables increases. When $N=300$ and $N=500$, adaLASSO always selects the correct model whereas Autometrics (Conservative) tends to include some irrelevant variables.

Concerning out-of-sample forecasting, for large values of $q$, even when adaLASSO finds the correct sparsity pattern, Autometrics (Conservative) presents better predictive performance. This is explained by the bias generated by the penalization term in adaLASSO that has stronger effect in RMSFE as $q$ increases. For small values of $q$ and $p<N$, adaLASSO and Autometrics (Conservative) have similar performance to the Oracle model.

A general conclusion is that, for a linear regression with orthogonal variables, the adaLASSO has superior performance in model selection than LASSO and Autometrics for almost every case $(N=100, N=300$ and $N=500)$. However, for small samples ( $N=50$ in our experiment), it is preferable to use Autometrics (Conservative).

In the application to psoriasis forecasting, Autometrics cannot handle all the genomic expressions as candidate variables in a feasible time. For that reason, the initial set of 54675 variables was reduced to a set of 870 genes. Results showed that LASSO and adaLASSO are much superior in predictive power than Autometrics. 


\section{Appendix}

Cross-block algorithm proposed in Hendry and Krolzig (2004) in the case where the number of candidate variables exceeds the number of observations in Autometrics:

1. dividing the set of variables into subsets (blocks), each of which contains less than half of the observations,

2. applying Autometrics model selection to each combination of the blocks (GUMs). The algorithm yields a terminal model for each GUM,

3. taking the union of the terminal models derived from each GUM, forming a new single union model.

4. If the number of variables in this model is less than the number of observations, model selection proceeds from this new union model (new unique GUM), otherwise, restarts the cross-block algorithm with the new set of variables.

\section{References}

Bowcock AM, Shannon W, Du F, Duncan J, Cao K, Aftergut K, et al. (2001). Insights into psoriasis and other inflammatory diseases from large-scale gene expression studies. Human Molecular Genetics, 10(17), 1793-1805.

Campos, J., D. F. Hendry, and H. M. Krolzig (2003). Consistent Model Selection by an Automatic Gets Approach. Oxford Bulletin of Economics and Statistics, 65, supplement, 803-819.

Correa da Rosa J, Kim J, Tian S, Tomalin LE, Krueger JG, Suárez-Fariñas M. (2017). Shrinking the Psoriasis Assessment Gap: Early Gene-Expression Profiling Accurately Predicts Response to Long-Term Treatment. The Journal of Investigative Dermatology, 137(2), 305-312.

Doornik, J. A. (2009). Autometrics. In J. L. Castle and N. Shephard (Eds.), The Methodology and Practice of Econometrics, pp. 88-122. Oxford University Press, Oxford.

Efron, B., Hastie, T., Johnstone, I. and Tibshirani, R. (2004). Least angle regression, The Annals of Statistics, 32(2), 407-499.

Fan, J. and Li, R. (2001). Variable selection via nonconcave penalized likelihood and its oracle properties. Journal of the American Statistical Association, 96, 1348-1360.

Friedman, J. H., Hastie, T., Tibshirani, R. (2010). Regularized Paths for Generalized Linear Models via Coordinate Descent. Journal of Statistical Software, 33(1). 
Harvey, D., Leybourne, S. and Newbold, P. (1997). Testing de equality of prediction mean squared errors. International Journal of Forcasting, 13, 281-291.

Hendry, D. F., and Krolzig, H-M. (1999). Improving on 'Data Mining Reconsidered' by K. D. Hoover and S. J. Perez. Econometrics Journal, 2, 202-219.

Hendry, D. F., and Krolzig, H.-M. (2004). Resolving three 'intractable' problems using a Gets approach. Unpublished paper, Economics Department, University of Oxford.

Hendry, D.F. and B. Nielsen (2007), Econometric Modeling: A Likelihood Approach. Princeton University Press.

Kim J, Nadella P, Kim DJ, Brodmerkel C, Correa da Rosa J, Krueger JG, SuárezFariñas M. (2015). Histological Stratification of Thick and Thin Plaque Psoriasis Explores Molecular Phenotypes with Clinical Implications. PLoS ONE, 10(7): e0132454.

Krolzig, H-M. and Hendry, D.F. (2001). Computer automation of general-to-specific model selection procedures. Journal of Economic Dynamics and Control, 25, 831866.

Medeiros, M. C. and Mendes, E. F. (2016). $\ell_{1}$-Regularization of High-dimensional Time-Series Models with Non-Gaussian and Heteroskedastic Innovations. Journal of Econometrics, 191, 255-271.

Meinshausen, N. and Yu, B. (2009). Lasso-type recovery of sparse representations for high dimensional data. The Annals of Statistics, 37, 246-270.

Perez-Amaral, T., Gallo, G. M. and White, H. (2003). A flexible tool for model building: the relevant transformation of the inputs network approach (RETINA). Oxford Bulletin of Economics and Statistics, Vol. 65, 821-838.

Suárez-Fariñas, M., Li, K., Fuentes-Duculan, J., Hayden, K., Brodmerkel, C., Krueger, J. G. (2012). Expanding the psoriasis disease profile: interrogation of the skin and serum of patients with moderate-to-severe psoriasis. The Journal of Investigative Dermatology, 132(11), 2552-64.

Tian, S., Krueger, J. G., Li, K., Jabbari, A., Brodmerkel, C., et al. (2012). MetaAnalysis Derived (MAD) Transcriptome of Psoriasis Defines the "Core" Pathogenesis of Disease. PLoS ONE 7(9): e44274.

Tian, S., Suárez-Fariñas, M. (2013). Multi-TGDR: a regularization method for multiclass classification in microarray experiments. PLoS ONE 8(11): e 78302.

Tibshirani, R. (1996). Regression shrinkage and selection via the Lasso. Journal of the Royal Statistical Society. Series B (Methodological) 58(1), 267-288.

Tibshirani, R. (2011). Regression shrinkage and selection via the lasso: a retrospective. JRSSB retrospective read paper, vol. 73, part 3, 273-282. 
Wang, H., Li, G. and Tsai, C. (2007). Regression coefficient and autoregressive order shrinkage and selection via the lasso. Journal of the Royal Statistical Society: Series B (Statistical Methodology), 69(1), 63-78.

White, H. (2006). Approximate nonlinear forecasting methods. In G. Elliott, C. W. J. Granger, and A. Timmermann (Eds.), Handbook of Economic Forecasting, Volume 1, pp. 459-512. Elsevier, Amsterdam.

Zhang, Y., Li, R. and Tsai, C.-L. (2010). Regularization parameter selections via generalized information criterion. Journal of the American Statistical Association, $105,312-323$.

Zhao, P. and Yu, B. (2006). On model consistency of lasso. Journal of Machine Learning Research, 7, 2541-2563.

Zou, H. (2006). The adaptive lasso and its oracle properties. Journal of the American Statistical Association, 101, 1418-1429.

Zou, H. and Hastie, T. (2005). Regularization and variable selection via the elastic net. Journal of Royal Statistical Society, Series B 67, 301-320.

Zou, H., Hastie, T. and Tibshirani, R. (2007). On the degrees of freedom of the lasso. Annals of Statistics, 35, 2173-2192. 